\title{
Optical focusing in scattering media with photoacoustic wavefront shaping (PAWS)
}

Puxiang Lai, Jian Wei Tay, Lidai Wang, Lihong V. Wang

Puxiang Lai, Jian Wei Tay, Lidai Wang, Lihong V. Wang, "Optical focusing in scattering media with photoacoustic wavefront shaping (PAWS)," Proc. SPIE 8943, Photons Plus Ultrasound: Imaging and Sensing 2014, 894318 (3 March 2014); doi: 10.1117/12.2036510

SPIE. Event: SPIE BiOS, 2014, San Francisco, California, United States 


\title{
Optical Focusing in Scattering Media with Photoacoustic Wavefront Shaping (PAWS)
}

\author{
Puxiang Lai ${ }^{\dagger}$, Jian Wei Tay ${ }^{\dagger}$, Lidai Wang ${ }^{\dagger}$, and Lihong V. Wang \\ (†'Equal contribution) \\ Washington University in St. Louis \\ Department of Biomedical Engineering, Optical Imaging Laboratory \\ Campus Box 1097, 1 Brookings Drive, St. Louis, Missouri 63130, USA
}

\begin{abstract}
Controllable light delivery to the region of interest is essential to biomedical optical imaging methods like photoacoustic microscopy. It is, however, challenging beyond superficial depths in biological tissue ( $\sim 1 \mathrm{~mm}$ beneath human skin) due to the strong scattering of light that scrambles the photon propagation paths. Recently, optical wavefront shaping has been proposed to modulate the incident light wavefront to compensate for the scattering-induced phase distortions, and consequentially, convey light optimally to a desired location behind or inside turbid media. To reach an optimum wavefront, a searching algorithm is usually required to optimize a feedback signal. In this work, we present our latest explorations, which use photoacoustic signals as the feedback to remotely and non-invasively guide the wavefront shaping process. Our method does not require direct optical access to the target region or the invasive embedding of fluorescence probes inside turbid media. Experimentally, we have demonstrated that diffuse light can be converged to the ultrasound focus by maximizing the amplitude of photoacoustic emissions from the intended absorbing site. Moreover, we show that wavefront-shaped light focusing can enhance existing optical imaging modalities like photoacoustic microscopy, in regard to signal-to-noise ratio, imaging depth, and potentially, resolution.
\end{abstract}

Keywords: optical focusing, wavefront shaping, photoacoustic imaging, light scattering, spatial light modulator, nonlinear photoacoustic signal, Grueneisen memory effect, optical speckle

\section{INTRODUCTION}

Light, in many ways, is an ideal choice to visualize biological structures, interrogate and control biological processes, and to diagnose and treat diseases. However, optical techniques lack spatial resolution in deep tissue due to the strong scattering of light from wavelength-scale refractive index changes in biological tissue [1]. As a result, when light is used alone, there is usually a trade-off between penetration depth and resolution; otherwise invasive procedures, such as embedded probes or guides, are required. Interdisciplinary methods have been developed to break this limitation, usually benefiting from the much weaker diffusivity of the mechanism other than light. Two types of ultrasound-mediated optical tomography-ultrasound-modulated optical tomography (UOT) [2] and photoacoustic imaging (PAI) [3, 4] -are examples. They have achieved optical contrast sensing with resolution provided by the externally applied ultrasound

Further author information: Puxiang Lai, plai@wustl.edu, 1-314-935 9587

Lihong V. Wang, lhwang@,wustl.edu, 1-314- 9356152

Photons Plus Ultrasound: Imaging and Sensing 2014, edited by Alexander A. Oraevsky, Lihong V. Wang, Proc. of SPIE Vol. 8943, 894318 - @ 2014 SPIE · CCC code: 1605-7422/14/\$18 · doi: 10.1117/12.2036510 
modulation [2] or the internally generated acoustic signal [3].

More recently, noticing that the appearance of random speckles formed by the propagation of coherent light in scattering media are actually deterministic within the speckle correlation time, researchers have started to explore the feasibility of optical focusing inside scattering media. For example, it has been shown that ultrasonically modulated/encoded light, as generated in UOT, can be time-reversed, and sent back to the scattering media, forming an optical focus at the ultrasonic focal position. This technique is usually called time-reversed ultrasonically encoded (TRUE) optical focusing [5-12], using either an analog (photorefractive material) or a digital (a digital camera plus a spatial light modulator) phase conjugation mirror to record and time-reverse the wavefront.

Another endeavor is optical wavefront shaping [13-23]. In this technique, the wavefront of an incident beam is shaped to compensate for the scattering-induced phase distortions, so that the scattered wavefronts interfere in phase at a predetermined location, and consequentially, form an optical focus. To reach the optimum wavefront compensation corresponding to a specific diffusivity pattern, a searching algorithm is usually employed to optimize a feedback signal. In this work, we present our preliminary explorations of using photoacoustic signals as the feedback to remotely and non-invasively guide the wavefront shaping process. This method, named photoacoustic wavefront shaping (PAWS), does not require either direct optical access to the target region or the invasive embedding of fluorescence probes inside scattering media. Experimentally, we show that diffuse light can be converged to the ultrasonic focus by maximizing the photoacoustic signal amplitude from the intended absorbing site.

\section{EXPERIEMENTAL SCHEMATIC AND PRINCIPLE}

As shown in Fig. 1 , the light from a pulsed laser source $(\lambda=570 \mathrm{~nm}$, pulsed width $=5 \mathrm{~ns}$, pulse energy $\approx 200 \mu \mathrm{J}$, pulse repetition rate $=1 \mathrm{kHz}$ ) was split into two beams by a half-wave plate ( $\lambda / 2$ in Fig. 1$)$ and a polarizing beam splitter (PBS). One beam, the vertically polarized portion reflected by the PBS, was detected by a photodiode to monitor the laser pulse energy fluctuations. The other beam, the horizontally polarized portion propagating through the PBS, was expanded and illuminated onto a spatial light modulator (SLM). The SLM has a full pixel resolution of $1920 \times 1080$. In our experiments, the SLM was divided into $40 \times 40$ independently controlled blocks, each with linearized phase shifts between 0 and $2 \pi$. The reflected beam from the SLM was then focused onto a tissue-mimicking ground glass diffuser using a microscopic objective lens, creating a randomized speckle pattern behind the diffuser. A piece of black tape (part of the sample in Fig. 1) was positioned in water behind the diffuser, and served as the absorptive material to generate photoacoustic (PA) signals under the pulsed optical illumination. A focused ultrasound transducer (central frequency $=5 \mathrm{MHz}$, lateral focal width $\approx$ $600 \mu \mathrm{m}$ ) was aligned to perceive PA emissions. The detected PA signals were amplified, digitized, and sent to a computer. The peak-to-peak amplitude of the acquired signals was quantified as the PA amplitudes, and was then normalized to the laser pulse energy. The measured amplitudes were then used as the feedback to guide the optimization of phase patterns on the SLM via a genetic algorithm $[18,22,24]$. Whenever a maximum PA amplitude was reached, the corresponding phase pattern on the SLM was considered the one that best compensated for the diffuser-induced scattering. Accordingly, an acoustic diffraction-limited optical focus at the transducer focal position would be expected. After the PAWS optimization, both the sample and the transducer were moved along the $X$ direction to create a pathway for the CCD camera to image the light spots illuminating the semi-transparent tape section when different phase patterns were displayed on the SLM. 


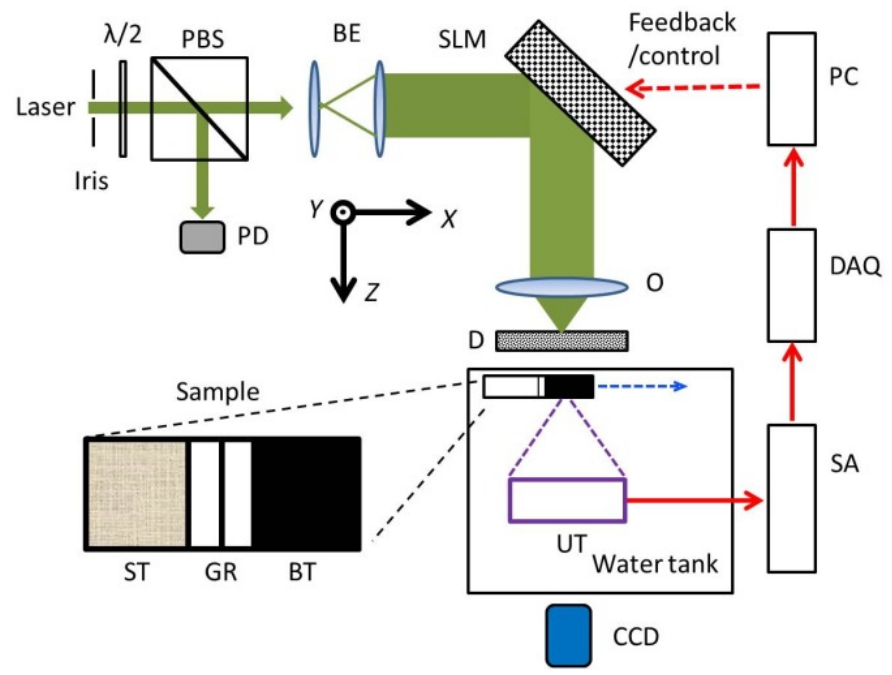

Fig. 1 Experimental schematic of PAWS. $\lambda / 2$, half-wave plate; BE, beam expander; CCD, CCD camera; D, ground glass diffuser; DAQ, data acquisition device; O, microscopic objective lens; PBS, polarizing beam splitter; PC, computer; SA, photoacoustic signal amplifier; SLM, spatial light modulator; UT, focused ultrasonic transducer; $X Y Z$, coordinate axes. The sample used to generate the photoacoustic signals during the optimization process is a piece of black tape (BT). After the PAWS, both the sample and the ultrasound transducer are moved along the $X$ direction, so that the CCD camera is able to image the optical beam patterns illuminated on the semi-transparent tape (ST). Between the BT and ST is a graphite rod (GR, a pencil core $500 \mu \mathrm{m}$ in diameter), which is used to calibrate the spatial scales of the beam pattern images.

\section{EXPERIMENTAL VALIDATIONS}
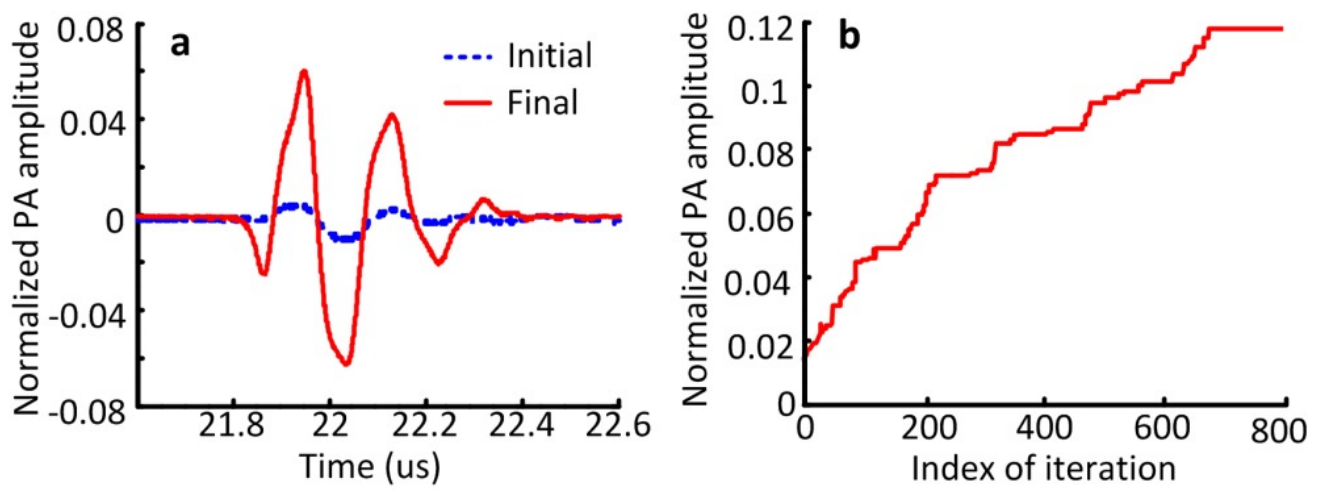

Fig. 2 (a) The initial and the final (optimized) PA signal before and after the PAWS optimization process. (b) Normalized PA amplitude versus index of iteration.

PAWS optical focusing was validated experimentally. First, a random phase pattern was displayed on the SLM. Accordingly, an initial PA signal (the blue, dashed curve in Fig. 2a) was recorded, after taking an average over 8 traces. As the genetic algorithm-based optimization proceeded, the normalized PA amplitude increased with the index of iteration (Fig. 2b). After $\sim 700$ iterations, the feedback plateaued. Therefore, the optimization was terminated after 800 iterations. 
The PA signal at $800^{\text {th }}$ iteration is shown in Fig. 2a. The signal enhancement factor between the final and the initial PA signal amplitude was $\mathrm{R} \approx 0.12 / 0.014 \approx 8.6$, indicating that optical energy within the acoustical focus was enhanced by $\sim 8.6$ times with PAWS.

The enhancement factor was confirmed from the comparison of imaged patterns shown in Fig. 3. As seen, light was diffused and no optical focus could be seen (Fig. 3a) when the random phase pattern was displayed. But with the optimized phase pattern, an optical focus was clearly seen (Fig. 3b), with a full width at half-maximum (FWHM) of $610 \mu$ m and 602 $\mu \mathrm{m}$ along the $X$ and $Y$ directions, respectively (Fig. 3c-d), agreeing with the lateral focal width of the ultrasonic transducer $(600 \mu \mathrm{m})$. This agreement demonstrates that the formed optical focus was acoustic-diffraction limited. Moreover, the peak-to-background ratio seen in Figs. $3 \mathrm{~b}$-d was around $1 / 0.12 \approx 8.3$, which was consistent with the 8.6 times enhancement in Fig. 2. It was also close to the theoretical enhancement factor estimated through the equation $\pi / 4 \times(\mathrm{N} / \mathrm{M})[7,13]$, where $\mathrm{N}=20 \times 20$ is the number of independently controlled blocks on the SLM, and M is the number of speckle grains within the acoustic focus, which was $\sim 119$ in the current setting.
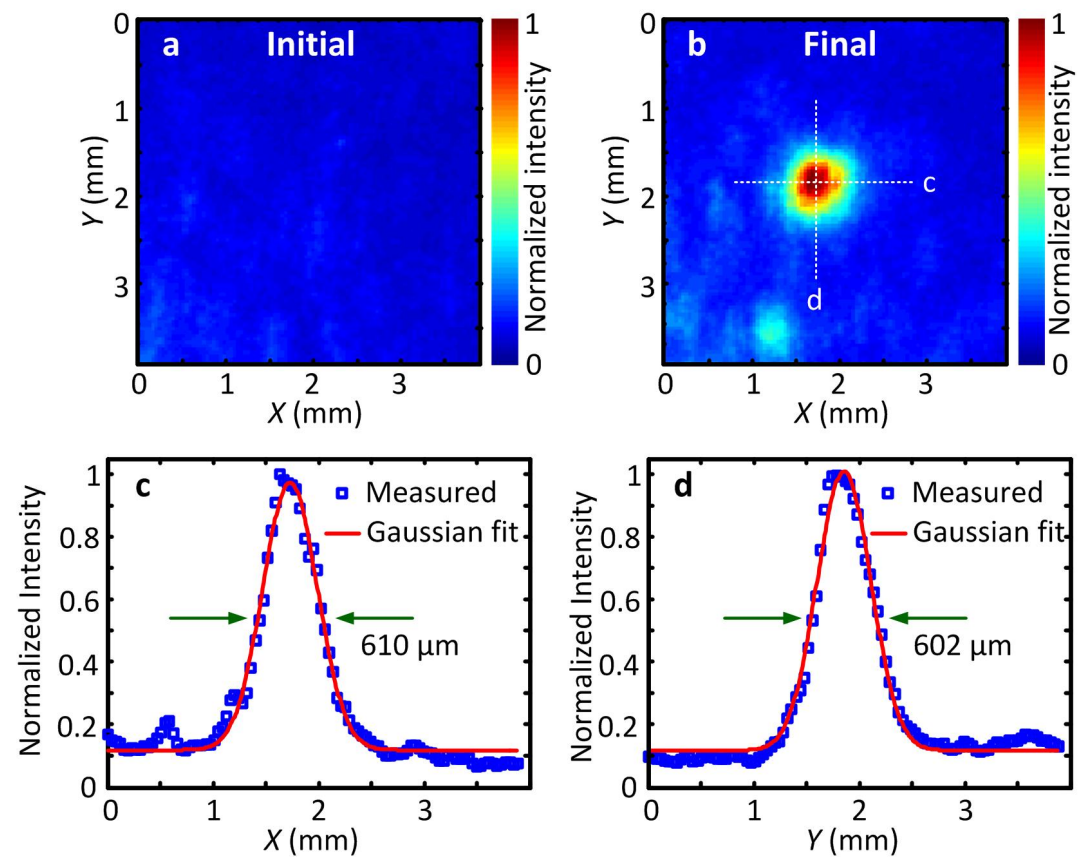

Fig. 3 The imaged beam pattern on the tape when a random (a) and the optimized phase pattern (b) were displayed on the SLM. Intensity distributions across the optical focus in (b) were shown in (c) and (d), along the $X$ and $Y$ axes respectively.

\section{SUMMARY AND FUTURE WORK}

We have shown in this work that one can use photoacoustic signals to guide optical wavefront shaping to achieve an acoustical diffraction-limited optical focus behind or inside scattering media. To break through this acoustic resolution limit, Conkey et al. [21] considered the non-uniform (Gaussian-like) spatial sensitivity profile of the ultrasonic transducer to discern and weight contributions to the final PA signals from each individual optical mode inside the ultrasonic focal region. Most recently, we proposed to use nonlinear photoacoustic signals, based on the Grueneisen memory effect, as 
feedback for iterative optimization, which we termed nonlinear PAWS [25]. In our pilot experiment, an optically diffraction-limited optical focusing with superior peak fluence gain ( $\sim 600$ times) was obtained in scattering media. Such an intense and highly confined optical focus in scattering media can benefit many micrometer-scale optical applications, especially if the optimization speed can be improved enough to enable biomedical applications in tissue, where optical speckles decorrelate fast due to physiological motions such as blood flow and aspiration.

\section{ACKNOWLEDGEMENTS}

This research is sponsored in part by the National Academies Keck Futures Initiative grant IS 13, National Institute of Health grants DP1 EB016986 (NIH Director's Pioneer Award), and R01 CA186567 (NIH Director's Transformative Research Award). L.W. has a financial interest in Microphotoacoustics, Inc. and Endra, Inc., which, however, did not support this work.

\section{REFERENCES}

[1] L. V. Wang, and H.-I. Wu, [Biomedical Optics: Principles and Imaging] John Wiley and Sons, Hoboken, New Jersey(2007).

[2] P. Lai, X. Xu, and L. V. Wang, "Ultrasound-modulated optical tomography at new depth," Journal of Biomedical Optics, 17(6), 066006 (2012).

[3] L. V. Wang, and S. Hu, "Photoacoustic Tomography: In Vivo Imaging from Organelles to Organs," Science, 335(6075), 1458-1462 (2012).

[4] M. Nasiriavanaki, J. Xia, H. Wan et al., "High-resolution photoacoustic tomography of resting-state functional connectivity in the mouse brain," Proceedings of the National Academy of Sciences, 111(1), 21-26 (2014).

[5] X. Xu, H. Liu, and L. V. Wang, "Time-reversed ultrasonically encoded optical focusing into scattering media," Nature Photonics, 5(3), 154-157 (2011).

[6] P. Lai, X. Xu, H. Liu et al., "Time-reversed ultrasonically encoded (TRUE) optical focusing in biological tissue," Journal of Biomedical Optics, 17(3), 030506 (2012).

[7] Y. M. Wang, B. Judkewitz, C. A. DiMarzio et al., "Deep-tissue focal fluorescence imaging with digitally time-reversed ultrasound-encoded light," Nat Commun, 3, 928 (2012).

[8] K. Si, R. Fiolka, and M. Cui, "Fluorescence imaging beyond the ballistic regime by ultrasound-pulse-guided digital phase conjugation,” Nat Photon, 6(10), 657-661 (2012).

[9] P. Lai, Y. Suzuki, X. Xu et al., "Focused fluorescence excitation with time-reversed ultrasonically encoded light and imaging in thick scattering media," Laser Physics Letters, 10(7), 075604 (2013).

[10] H. Liu, X. Xu, P. Lai et al., "Time-reversed ultrasonically encoded (TRUE) optical focusing into tissue-mimicking media with optical thickness up to 70, J Journal of Biomedical Optics, 16(8), 086009 (2011).

[11] P. Lai, X. Xu, H. Liu et al., "Reflection-mode time-reversed ultrasonically encoded (TRUE) optical focusing into turbid media," Journal of Biomedical Optics, 16(8), 080505 (2011).

[12] Y. Suzuki, X. Xu, P. Lai et al., "Energy enhancement in time-reversed ultrasonically encoded optical focusing using a photorefractive polymer," Journal of Biomedical Optics, 17(8), 080507 (2012).

[13] I. M. Vellekoop, and A. P. Mosk, "Focusing coherent light through opaque strongly scattering media," Opt. Lett., 32(16), 2309-2311 (2007). 
[14] A. P. Mosk, A. Lagendijk, G. Lerosey et al., "Controlling waves in space and time for imaging and focusing in complex media," Nat Photon, 6(5), 283-292 (2012).

[15] S. Popoff, G. Lerosey, M. Fink et al., "Image transmission through an opaque material," Nature Communications, 1, 81-85 (2010).

[16] S. M. Popoff, G. Lerosey, R. Carminati et al., "Measuring the Transmission Matrix in Optics: An Approach to the Study and Control of Light Propagation in Disordered Media," Physical Review Letters, 104(10), 100601 (2010).

[17] T. Chaigne, O. Katz, A. C. Boccara et al., "Controlling light in scattering media noninvasively using the photo-acoustic transmission-matrix," Nature Photonics, 8(1), 58-64 (2013).

[18] D. B. Conkey, A. N. Brown, A. M. Caravaca-Aguirre et al., "Genetic algorithm optimization for focusing through turbid media in noisy environments," Opt. Express, 20(5), 4840-4849 (2012).

[19] D. B. Conkey, A. M. Caravaca-Aguirre, and R. Piestun, "High-speed scattering medium characterization with application to focusing light through turbid media," Opt. Express, 20(2), 1733-1740 (2012).

[20] F. Kong, R. H. Silverman, L. Liu et al., "Photoacoustic-guided convergence of light through optically diffusive media," Opt. Lett., 36(11), 2053-2055 (2011).

[21] D. B. Conkey, A. M. Caravaca-Aguirre, J. D. Dove et al., "Super-resolution photoacoustic imaging through a scattering wall," arXiv, eprint arXiv:1310.5736 (2013).

[22] J. W. Tay, P. Lai, Y. Suzuki et al., "Ultrasonically encoded wavefront shaping for focusing into random media," Scientific Reports, 4, 3918 (2014).

[23] A. M. Caravaca-Aguirre, D. B. Conkey, J. D. Dove et al., "High contrast three-dimensional photoacoustic imaging through scattering media by localized optical fluence enhancement," Optics Express, 21(22), 26671-26676 (2013).

[24] O. Katz, E. Small, Y. Broomberg et al., "Focusing and compression of ultrashort pulses through scattering media," Nature Photonics, 5, 372-377 (2011).

[25] P. Lai, L. Wang, J. W. Tay et al., "Nonlinear photoacoustic wavefront shaping (PAWS) for single speckle-grain optical focusing in scattering media," arXiv, eprint, arXiv:1402.0816 (2014). 